\title{
A Fatal Case of Clostridium difficile Enterocolitis following Diverting Loop Ileostomy Reversal
}

\author{
Cybele Arsan,1,2 Omar Y Gonzalez, ${ }^{1,3}$ and Thomas L. Gillespie ${ }^{1,4}$
}

\begin{abstract}
Background: Clostridium difficile infection (CDI) is one of the most common hospital-acquired infections (HAI). Fulminant colitis is a severe form of the disease and is considered a surgical emergency. A total colectomy is the current standard of care for patients with this diagnosis. A new emerging procedure, diverting loop ileostomy with colonic lavage, has shown to have less morbidity, allowing preservation of the native colon. Methods: We present a patient who developed sepsis secondary to fulminant CDI. He received treatment with diverting loop ileostomy and colonic lavage in addition to antimicrobial agents. Seven months later, disease free, he underwent ileostomy reversal but then developed recurrent toxic megacolon and enteritis secondary to CDI. Conclusion: Even though diverting loop ileostomy with colonic lavage in the setting of a complicated infection seems to have initial benefits, additional studies are needed to address long-term morbidity/mortality.
\end{abstract}

$C$ LOSTRIDIUM DIFFICILE INFECTION is one of the most common HAIs. It usually results from antibiotic exposure. Clindamycin and fluoroquinolones have been linked to the majority of cases [1]. Antibiotics cause a disruption of the natural colonic flora allowing overgrowth of $C$. difficile, a spore forming, anaerobic, gram-positive bacilli that produces two types of toxins: Toxin A (enterotoxin) and toxin B (cytotoxin). These toxins bind to intestinal epithelial cell receptors, which causes inflammation and diarrhea. Several risk factors such as inflammatory bowel disease (IBD), hospitalization, and advanced age make individuals more susceptible to suffer from a primary infection by $C$. difficile.

The relapse rate of CDI is $20 \%-30 \%$, and is observed mainly among patients older than 75 years with the presence of at least 10 unformed stools (over $24 \mathrm{~h}$ ) or with a serum creatinine level of at least $1.2 \mathrm{mg} / \mathrm{dL}$ [2]. The most severe form of CDI is fulminant colitis, with or without toxic megacolon, with a mortality rate of $75 \%$ or greater. Treatment of the infection varies depending on the severity of the disease but it is recommended to start by discontinuing the administration of the offending antibiotic, if possible. Severe CDI is treated usually with intravenous (IV) metronidazole with vancomycin via oral (PO) or rectal-enema (PR) route; the most severe cases often require surgery [3]. The standard surgical approach is total colectomy. However, this invasive procedure has high morbidity and mortality in critically ill patients. Neal et al. [4] found that among 42 patients who underwent a diverting loop ileostomy and colonic lavage, a mortality rate of $19 \%$ (compared with $50 \%$ using historical data) was observed, allowing them to preserve their native colon for future re-anastomosis.

\section{Methods}

From 2012 to 2015, we found a single case of $C$. difficile enterocolitis following a diverting loop ileostomy reversal at our institution, a tertiary care hospital and level 1 trauma center in Phoenix, Arizona.

We performed a literature review using PubMed from 2005-2015. Clinical and laboratory standard institute guidelines were used for toxin detection (glutamate dehydrogenase enzyme immunoassay $[\mathrm{GDH}]$ ) and real-time polymerase chain reaction. Intestinal tissue gold culture with reflex cytotoxin cell assay and minimal inhibitory concentration (MIC) for metronidazole and vancomycin were performed by Associated Regional and University Pathologists (ARUP). For pathology, the small bowel specimen was sent in formalin, fragmented upon sectioning, and routine hematoxylin and eosin (H\&E) staining was performed. Surgical specimens were

\footnotetext{
${ }^{1}$ Saint Joseph's Hospital and Medical Center, Phoenix, Arizona.

${ }^{2}$ Creighton University School of Medicine, Phoenix, Arizona.

${ }^{3}$ Department of Medicine, Infectious Disease, ${ }^{4}$ Department of General Surgery, St. Joseph's Hospital and Medical Center, Phoenix, Arizona.
}

(C) Cybele Arsan et al. 2016; Published by Mary Ann Liebert, Inc. This Open Access article is distributed under the terms of the Creative Commons License (http://creativecommons.org/licenses/by/4.0), which permits unrestricted use, distribution, and reproduction in any medium, provided the original work is properly credited. 


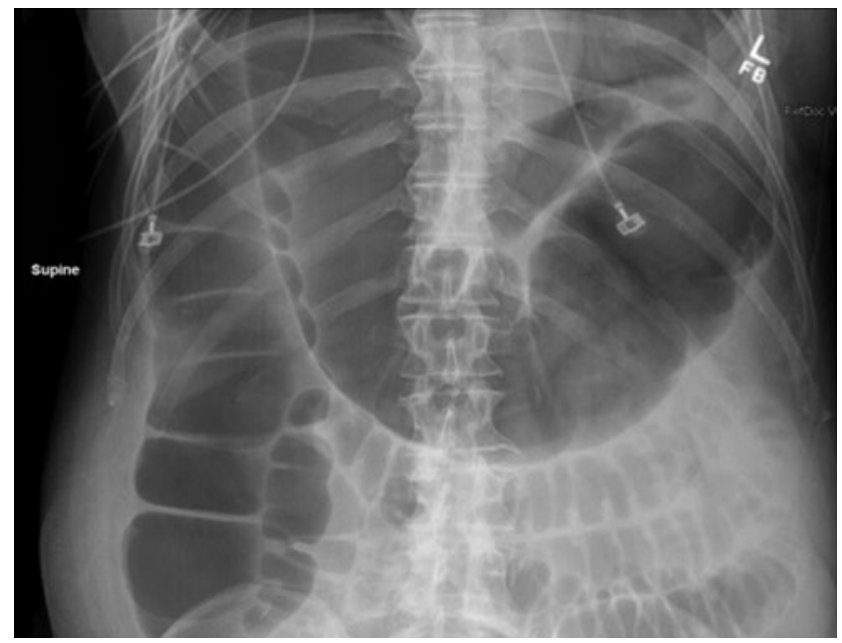

FIG. 1. Abdominal radiograph. Megacolon findings including extremely dilated colonic loops $(10.7 \mathrm{~cm})$ and loss of Hautra.

submitted and analyzed by the department of pathology under standard protocol.

\section{Case Presentation}

A 64-year old Caucasian male with a past medical history of hypertension, hyperlipidemia, stroke, and pulmonary embolism presented with fulminant $C$. difficile colitis. He underwent a diverting loop ileostomy. He initially received IV metronidazole, $\mathrm{PO}$ and PR vancomycin, and subsequently post-surgery, he was treated with $1,000 \mathrm{mg} \mathrm{PO}$ vancomycin every $6 \mathrm{~h}$ and $1,000 \mathrm{mg}$ colonic lavage vancomycin every $6 \mathrm{~h}$ via a catheter placed inside the distal portion of the ostomy. He improved and was discharged to a skilled nursing facility (SNF). At his SNF he completed a 5-mo taper of PO and colonic lavage with vancomycin; clinically he was disease free upon completion of his therapy. Seven months later he

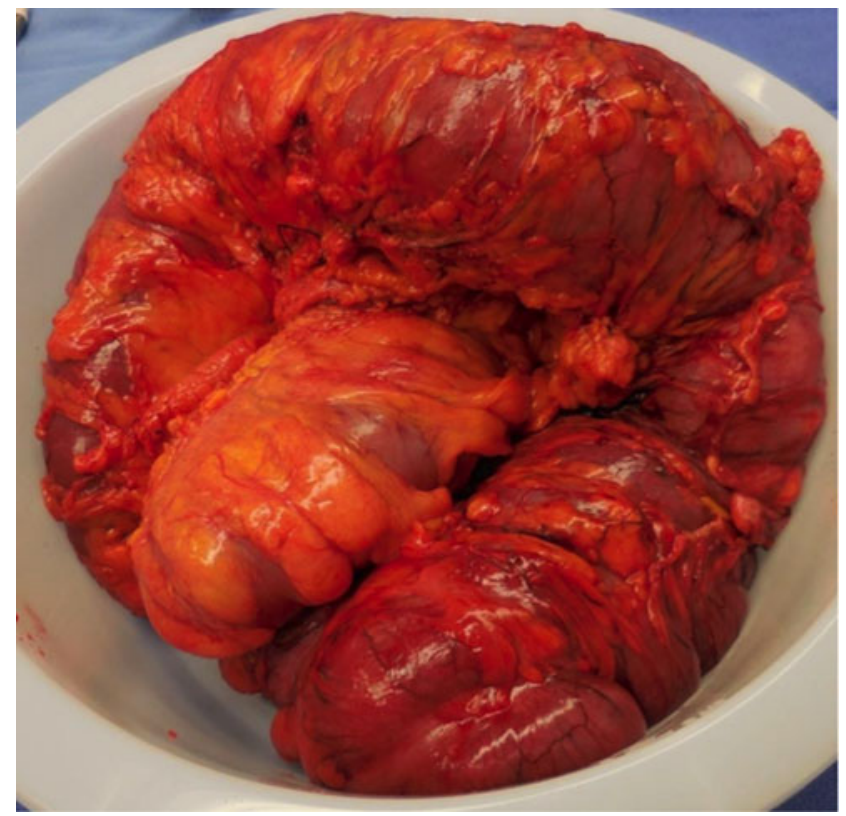

FIG. 2. Toxic megacolon immediately after resection in the operating room, showing signs of severe inflammation but no perforation or necrosis.

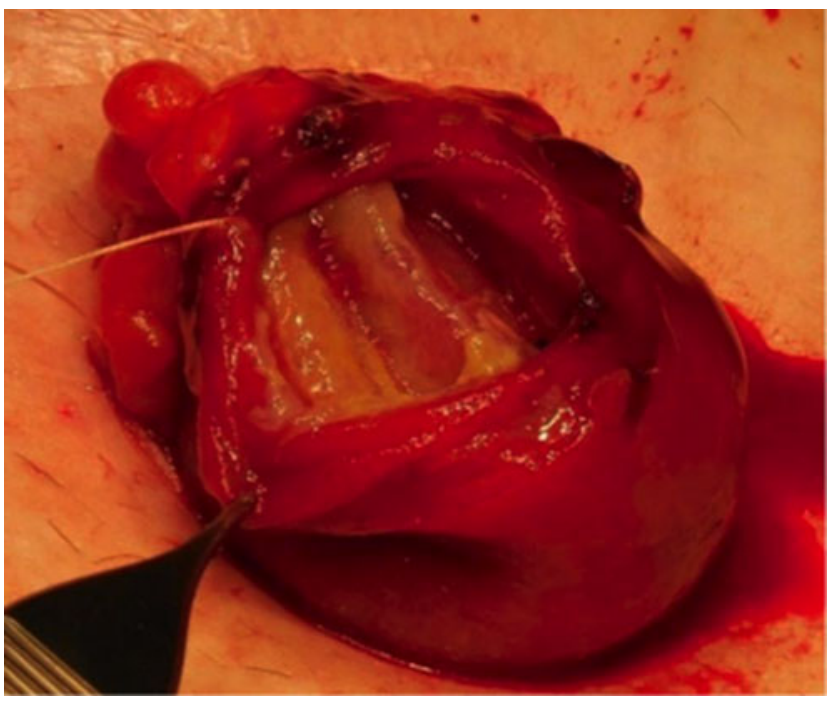

FIG. 3. Ileum section showing pseudomembranous findings.

presented for reversal of the ileostomy. A pre-operative colonoscopy showed no abnormalities. He received one dose of cefoxitin and underwent an ileostomy reversal surgery with no complications. On post-operative day (POD) 4 he developed diarrhea and was started on PO vancomycin; stool samples were positive for $C$. difficile. On POD 5 he became septic. Abdominal radiograph (Fig. 1) demonstrated findings consistent with toxic megacolon. He underwent exploratory laparotomy and total colectomy with ileostomy placement. A large, distended colon was removed (Fig. 2) but showed no evidence of perforation. On the distal ileal lumen of his ileostomy we found the presence of membranes (Fig. 3), which were sent to pathology and microbiology. Pathology reported presence of neutrophils and fibrin, which were consistent with pseudomembranous disease of the small bowel (Fig. 4). Microbiology yielded a positive culture for C. difficile. Additionally, stool samples from the ostomy showed a positive test for $C$. difficile toxin.

The patient was transferred to the intensive care unit (ICU) given his critical condition. He developed septic shock and was treated aggressively; he received broad spectrum

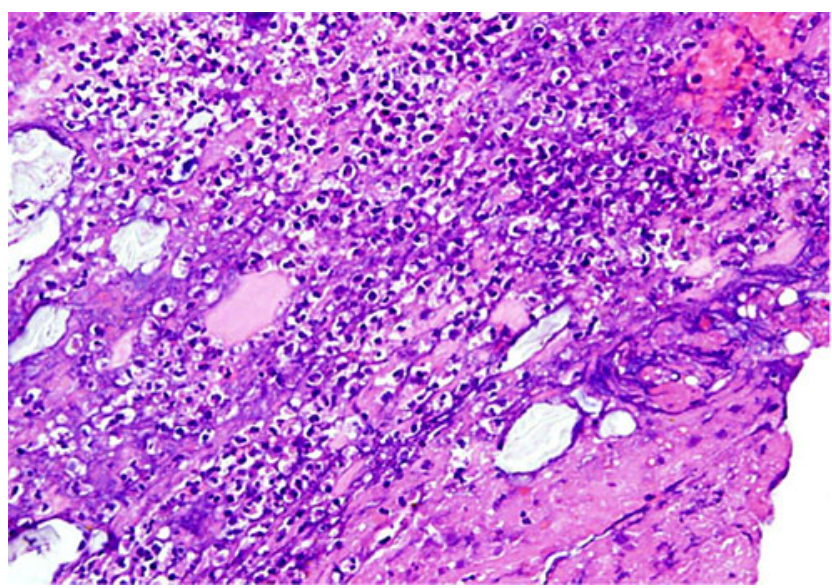

FIG. 4. Pathology microscopic view of small bowel membrane sample showing juxtaposition of neutrophils and fibrin consistent with pseudomembranous enteritis. 
antibiotics including vancomcycin enema and PO (via nasogastric tube) meropenem IV, metronidazole IV, and micafungin IV. He continued to deteriorate and meropenem was changed to tigecycline. Due to his poor prognosis despite treatment, his medical power of attorney withdrew medical care and the patient died.

\section{Discussion}

Despite its prevalence, severe $C$. difficile colitis is a difficult disease to manage. Even though a diverting loop ileostomy with colonic lavage is a promising initial strategy, morbidity following re-anastamosis has not been adequately investigated. In our case, it was complicated with toxic megacolon with pseudomembranous enteritis. This outcome might have been because of exposure to antibiotic prophylaxis (cefoxitin) and a hypervirulent strain described previously in the literature [5]. C. difficile NAP1/027 has been shown to increase toxin production, colonizing the small bowel more frequently, and also having a greater case-fatality ratio. Unfortunately, our patient's isolate was not genotyped. Our patient was treated with metronidazole and vancomycin, and he was considered cured before the re-anastomosis. Other treatment modalities now exist for relapses, i.e., fidaxomicin; that has been proposed as an alternative for refractory cases [6]. Another option is the use of fecal colonic microbiota transplantation, a modality that is increasingly used for colonic flora depleted patients, and in patients with a diverting ileostomy. It is a relatively simple procedure.

In retrospect, with no data to support, a pre-emptive therapy to prevent a relapse should have been done. It is not known if pre-treating the patient with metronidazole/vancomycin versus fidaxomicin versus fecal colonic transplant could have had a positive outcome. Moreover, withholding any other antibiotic such as the routine use for prophylaxis prior to surgery could have had a positive impact on his final outcome. This management could also have been repeated after the surgery with the theoretical goal of improving the outcome.

A future approach to severe $C$. difficile colitis is the use of intravenous immunoglobulin (IVIG) monoclonal antibody against toxins. This therapy is not yet available but shows promising results in decreasing the recurrence rates of $C$. difficile colitis. Anion-binding resins (ABR) have been described to bind the $C$. difficile toxins and were therefore a good prospect for adjuvant therapy ${ }^{1}$. However, their use is controversial for two reasons. First, ABR also binds vancomycin so administration of both agents needs to be timed 2$4 \mathrm{~h}$ apart. Also, ABRs also decrease cholesterol reabsorption by the colon and therefore increase diarrhea, which leads to more aggressive supportive care.

\section{Conclusion}

Diverting loop ileostomy with colonic lavage is a promising procedure that successfully may get the patient through their initial presentation of fulminant $C$. difficile colitis, however, the proof of success of this strategy can only be measured by the final outcome. More investigation about the pre- and postoperative management could improve outcomes further. The role of withholding antibiotic prophylaxis, pre-emptive therapy, fecal bacterio-therapy, monoclonal antibodies against C. difficile toxins, and anion-binding resins should be studied in the setting of ileostomy reversal.

\section{Author Disclosure Statement}

The authors declare there are no conflicts of interest.

\section{References}

1. Cohen SH, Gerding DN, Johnson S, et al. Clinical practice guidelines for Clostridium difficile infection in adults: 2010 update by the society for health care epidemiology of America (SHEA) and the infectious diseases society of America (IDSA). Infect Control Hosp Epidemiol. 2010;31:431-455.

2. D'Agostino RB Sr, Collins SH, Pencina KM, et al. Risk estimation for recurrent Clostridium difficile infection based on clinical factors. Clin Infect Dis 2014;58:1386.

3. Cohen SH, Gerding DN, Johnson S, et al. Clinical practice guidelines for Clostridium difficile infection in adults: 2010 update by the society for health care epidemiology of America (SHEA) and the infectious diseases society of America (IDSA). Infect Control Hosp Epidemiol. 2010;31:431-455.

4. Neal MD, Alverdy JC, Hall DE, et al. Diverting loop ileostomy and colonic lavage: An alternative to total abdominal colectomy for the treatment of severe, complicated Clostridium difficile associated disease. 2011;254:423.

5. Cohen SH, Gerding DN, Johnson S, et al. Clinical practice guidelines for Clostridium difficile infection in adults: 2010 update by the society for health care epidemiology of America (SHEA) and the infectious diseases society of America (IDSA). Infect Control Hosp Epidemiol. 2010;31:431-455.

6. Eyre W, Babakhani F, Griffiths D, et al. Whole-genome sequencing demonstrates that fidaxomicin is superior to vancomycin for preventing reinfection and relapse of infection with Clostridium difficile. J Infect Dis 2014;209:1446-1451.

7. Cohen SH, Gerding DN, Johnson S, et al. Clinical practice guidelines for Clostridium difficile infection in adults: 2010 update by the society for health care epidemiology of America (SHEA) and the infectious diseases society of America (IDSA). Infect Control Hosp Epidemiol. 2010;31:431-455.

Address correspondence to: Dr. Cybele Arsan CUSOM Phoenix Regional Campus St. Joseph's Hospital and Medical Center 350 West Thomas Road Phoenix, AZ 85013

E-mail: Cybelearsan@creighton.edu

$\begin{aligned} & \text { Abbreviations Used } \\ & \text { CDI }=\text { Clostridium difficile infection } \\ & \mathrm{HAI}=\text { hospital-acquired infections } \\ & \mathrm{IBD}=\text { inflammatory bowel disease } \\ & \mathrm{IV}=\text { intravenous } \\ & \mathrm{GDH}=\text { glutamate dehydrogenase enzyme immunoassay } \\ & \mathrm{MIC}=\text { minimal inhibitory concentration } \\ & \mathrm{SNF}=\text { skill nursing facility } \\ & \mathrm{POD}=\text { post-operative day } \\ & \mathrm{ICU}=\text { intensive care unit } \\ & \mathrm{IVIG}=\text { intravenous immunoglobulin } \\ & \mathrm{ABR}=\text { anion-binding resins }\end{aligned}$

Cite this article as: Arsan C, Gonzalez OY, Gillespie TL (2016) A Fatal Case of Clostridium difficile Enterocolitis following Diverting Loop Ileostomy Reversal, Surgical Infections Case Reports 1:1, 16-18, DOI: 10.1089/ crsi.2016.006. 